\title{
Comportamento de espécies herbáceas em misturas de solo com diferentes graus de contaminação com metais pesados(1)
}

\begin{abstract}
Marco Aurélio Carbone Carneiro(2), José Oswaldo Siqueira ${ }^{(3)}$ e Fátima Maria de Souza Moreira ${ }^{(3)}$
Resumo - O objetivo deste trabalho foi avaliar, em casa de vegetação, o comportamento de espécies herbáceas em relação ao excesso de $\mathrm{Cd}$ e $\mathrm{Zn}$ no solo. O gradiente de contaminação foi estabelecido a partir de mistura de solo contaminado com solo sem contaminação em diferentes proporções. As sementes foram semeadas em tubetes contendo $250 \mathrm{~mL}$ de solo, e após 90 dias as plantas foram colhidas e avaliadas. A maioria das espécies apresentou redução no crescimento com aumento da contaminação do solo e elevadas concentrações de Cd e Zn na matéria seca da parte aérea (MSPA), na mistura com 15\% de solo contaminado. A espécie Pffafia sp. mostrou-se tolerante à contaminação, crescendo em misturas de solo contendo até $90 \mathrm{mg} \mathrm{kg}^{-1}$ de Cd e $1.450 \mathrm{mg} \mathrm{kg}^{-1}$ de zinco. Além disso, apresentou concentração superior a $100 \mathrm{mg} \mathrm{kg}^{-1}$ de Cd na MSPA, sendo considerada hiperacumuladora desse metal. Sida glaziovii, Bidens pilosa, Rhynchelytrum repens, Cenchrus echinatus e Nicandra physaloides, por sua vez, foram severamente afetadas pela contaminação, ao contrário de Trifolium repens, Euchlaena mexicana, Cynodon dactylon, Avena strigosa, Cenchrus ciliares e Cyperus sp. que apresentaram crescimento satisfatório. As espécies avaliadas mostram-se promissoras para estudos adicionais sobre a reabilitação de áreas contaminadas com metais pesados.
\end{abstract}

Termos para indexação: cádmio, zinco, poluente, toxicidade do solo.

\section{Behavior of herbaceous species in soil mixes with different degree of contamination with heavy metal}

\begin{abstract}
In a study developed under greenhouse conditions using soil mixes with different degrees of contamination with heavy metal the behavior of herbaceous species in relation to the excess of $\mathrm{Cd}$ and $\mathrm{Zn}$ in the soil was evaluated. Gradient of soil contamination was established through soil mixes of heavy metal contaminated with uncontaminated soils at different proportions. Seeds of different species and origins were sowed in $250 \mathrm{~mL}$ plastic tubes and plants allowed to grow for 90 days when they were harvested and assessed. It was found a similar behavior for most species that exhibited growth depressions due to increasing soil contamination. At soil mix with $15 \%$ of contamination soil, plants showed high concentration of $\mathrm{Cd}$ and $\mathrm{Zn}$. In contrast to those species Pffafia sp. exhibited high tolerance to contamination, growing quite abundantly in soil mix with 90 and $1,450 \mathrm{mg} \mathrm{kg}^{-1}$ of $\mathrm{Cd}$ and $\mathrm{Zn}$, respectively. Moreover, this species showed Cd concentration higher than $100 \mathrm{mg} \mathrm{kg}^{-1}$, being considered $\mathrm{Cd}$ hiperacumulator. Sida glaziovii, Bidens pilosa, Rhynchelytrum repens, Cenchrus echinatus and Nicandra physaloides were severely affected by the contaminants. While the species Trifolium repens, Euchlaena mexicana, Cynodon dactylon, Avena strigosa, Cenchrus ciliares and Cyperus sp. presented adequate growth. These species are promising for further studies on rehabilitation of heavy metal degraded land.
\end{abstract}

Index terms: cadmium, zinc, pollutants, soil toxicity.

(1) Aceito para publicação em 24 de julho de 2002 .

Trabalho financiado pelo convênio Companhia Mineira de Metais, Faepe e Fapemig.

(2) Universidade Federal de Goiás, Campus Avançado de Jataí, Centro de Ciências Agrárias, Rod. BR 364, km 192, CEP 75800-000 Jataí, GO. E-mail: mcarbone@jatai.ufg.br

(3) Universidade Federal de Lavras, Dep. de Ciências do Solo, Caixa Postal 37, CEP 37200-000 Lavras, MG. E-mail: siqueira@ufla.br, fmoreira@ufla.br

\section{Introdução}

A poluição do solo com metais pesados, pela intensificação das atividades industriais, agrícolas e urbanização, é um problema crescente e responsável por sérios impactos ao ambiente (Sengupta, 1993). Uma das atividades que mais contribuem para a poluição do solo com esses agentes é a mineração e a indústria metalúrgica, principalmente a de metais não 
ferrosos, que ao promoverem a extração de alguns metais, produzem grandes quantidades de rejeitos, muitas vezes com elevados teores de $\mathrm{Ni}, \mathrm{Cr}, \mathrm{Cu}, \mathrm{Pb}$, Cd e Zn, que afetam severamente a vegetação (Baker et al., 1994) e as atividades biológicas do solo, como respiração e decomposição, no local e em áreas adjacentes (Vangronsveld et al., 1997). A destruição da cobertura vegetal em áreas contaminadas agrava a degradação do solo, promovendo erosão hídrica e eólica e a lixiviação dos contaminantes para o lençol freático, desencadeando progressivo grau de contaminação de outras áreas. Essas áreas precisam ser reabilitadas, e para tal exigem estudos de diversas naturezas sobre o solo, a vegetação e a água (Cunningham et al., 1996).

Uma das estratégias de reabilitação in situ dessas áreas é a fitorremediação, que consiste na introdução de vegetação no solo (Baker et al., 1994) mediante diferentes técnicas como a fitoestabilização (Accioly \& Siqueira, 2000). Na fitoestabilização utilizam-se plantas e agentes amenizantes do solo, como calcário (Accioly, 2001), para remover, imobilizar ou tornar os contaminantes menos nocivos ao ecossistema. Um dos requisitos básicos para o sucesso de qualquer técnica de revegetação é encontrar plantas tolerantes aos contaminantes. Estudo sobre a viabilidade de espécies arbóreas para a reabilitação de áreas contaminadas por metais pesados tem merecido destaque por causa da maior imobilização destes na biomassa vegetal por períodos mais longos (Eltrop et al., 1991). No entanto, existem evidências de que as plantas herbáceas possuem maior tolerância ao excesso de metais no solo do que as arbóreas (Baker, 1987).

Estudos realizados em regiões temperadas com espécies de plantas tolerantes a metais pesados, como Agrostis tenus, Agrotis stolonifera, Festuca rubra e Brassica sp., mostraram o sucesso do estabelecimento da vegetação herbácea nessas áreas (Ebbs \& Kochian, 1998; Salt et al., 1998). Plantas tolerantes e que acumulam grandes quantidades de metais em seus tecidos são designadas hiperacumuladoras (Baker, 1987), por apresentarem concentrações superiores a $100 \mathrm{mg} \mathrm{kg}^{-1}$ de Cd e de $10.000 \mathrm{mg} \mathrm{kg}^{-1}$ de Zn na matéria seca, como ocorre com algumas brássicas (Brassica juncea, B. rapa e Thlaspi caerulenscens) cujas concentrações de $\mathrm{Cd}$ e Zn atingem, respectivamente, 175 e $52.000 \mathrm{mg} \mathrm{kg}^{-1}$ (Salt et al., 1995; Cunningham \& Ow, 1996). Carneiro et al. (2001) observaram que a semeadura conjunta de várias espécies de plantas herbáceas, incluindo uma espécie de brássica tolerante ao $\mathrm{Cd}$ e ao $\mathrm{Zn}$, aumentou a possibilidade de sucesso da revegetação em solos contaminados pelo excesso desses metais, possivelmente pela maior absorção de $\mathrm{Cd}$ e $\mathrm{Zn}$ pela brássica.

Estudos com esse enfoque não são conhecidos nas condições brasileiras, onde a contaminação do solo e a existência de áreas degradadas pelo excesso de metais são cada vez mais numerosas, portanto, o conhecimento do comportamento de espécies herbáceas com potenciais para fitorremediação dessas áreas se reveste da maior importância.

O objetivo deste trabalho foi avaliar o comportamento de espécies herbáceas em relação ao excesso de $\mathrm{Cd}$ e $\mathrm{Zn}$ no solo.

\section{Material e Métodos}

O estudo foi realizado em casa de vegetação do Departamento de Ciência do Solo da Universidade Federal de Lavras (Ufla), utilizando solo contaminado com vários metais pesados coletado em área de rejeito da Companhia Mineira de Metais (CMM) situada em Três Marias, MG. O solo foi classificado como antropogênico, pois não apresentava distinção entre os horizontes pedogenéticos naturais, por causa da grande deposição de rejeitos industriais. A análise química revelou os seguintes resultados: (em mg kg-1 de solo) 18.600 de Zn; 140 de Cd; 450 de Cu; $410 \mathrm{de} \mathrm{Pb} ; 35$ de $\mathrm{Ni}$ e 1 de $\mathrm{Cr}$, extraído por água régia (Forster, 1995). Foram empregadas misturas de solo contendo $0 \% ; 7,5 \% ; 15 \% ; 30 \% ; 45 \%$ e $60 \%(\mathrm{v} / \mathrm{v})$ de solo contaminado com metais pesados, nos quais foram semeadas 31 espécies herbáceas (Tabela 1), em delineamento inteiramente casualizado, com cinco repetições. As espécies utilizadas foram selecionadas pela disponibilidade de sementes. Na diluição empregou-se Latossolo Vermelho ácrico típico, coletado $(0-20 \mathrm{~cm})$ no campus da Ufla. Antes de proceder a mistura, o solo recebeu calcário dolomítico para elevar a saturação de base para $70 \%$, incubando-o por 15 dias. Após esse período, aplicaram-se $200 \mathrm{mg} \mathrm{kg}^{-1} \mathrm{de} \mathrm{P}$ (superfosfato simples) e $50 \mathrm{mg} \mathrm{kg}^{-1} \mathrm{de} \mathrm{N}$ (nitrato de amônio), procedendo as misturas de modo a obter as proporções de solo contaminado desejadas. Com exceção dos teores de metais pesados, as misturas variaram muito pouco quanto às características químicas, apre- 
sentando, em média, $\mathrm{pH}$ em água, 6,5; $\mathrm{P}$, $77 \mathrm{mg} \mathrm{dm}^{-3}$; K, $208 \mathrm{mg} \mathrm{dm}^{-3}$; Ca, 5,3 mmol $_{\mathrm{c}} \mathrm{dm}^{-3} ; \mathrm{Mg}$, 3,2 $\mathrm{mmol}_{\mathrm{c}} \mathrm{dm}^{-3} \mathrm{e} \mathrm{V}, 78 \%$. As concentrações de $\mathrm{Zn}$ e $\mathrm{Cd}$ nas misturas, assim como as regressões para os teores desses elementos nas diferentes misturas de solo, encontram-se na Tabela 2 .

As misturas preparadas foram acondicionadas em tubetes com capacidade de $250 \mathrm{~mL}$ de solo, nos quais foram semeadas as diferentes espécies. Cada tubete recebeu de três a cinco sementes, com posterior desbaste para uma planta. O experimento foi conduzido por 90 dias e durante esse período os tubetes foram irrigados diariamente com água deionizada de modo a manter a umidade em nível adequado ao desenvolvimento das plantas (aproximadamente $60 \%$ do volume total de poros). As plantas foram colhidas, separadas em parte aérea e raízes, lavadas com água destilada e secadas em estufa com circulação forçada a $70^{\circ} \mathrm{C}$ até peso constante, obtendo-se o peso da matéria seca da parte aérea (MSPA) e das raízes (MSR). Esses materiais foram moídos em moinho tipo Willey para determinação dos teores de $\mathrm{Cd}$ e $\mathrm{Zn}$ nos extratos dos tecidos (Hunter, 1975) por espectrofotometria de absorção atômica. Consideraram-se apenas esses dois metais por apresentarem as maiores quantidades no solo em estudo, além de exercerem efeitos fitotóxicos mais acentuados nas plantas.

Tabela 1. Nome comum e científico, família e procedência das espécies estudadas.

\begin{tabular}{|c|c|c|c|}
\hline Nome comum & Nome científico & Família & Procedência \\
\hline Picão-preto & Bidens pilosa $\mathrm{L}$. & Compositae & Ufla $^{(1)}$ \\
\hline Guanxuma & Sida glaziovii K. Schum & Malvaceae & $\mathrm{Ufla}^{(1)}$ \\
\hline Lobeira & Nicandra physaloides Gaertn. & Solanaceae & $\mathrm{Ufla}^{(1)}$ \\
\hline Amendoim-bravo & Arachis pintoi $\mathrm{L}$. & Leguminosae & Matsuda $^{(2)}$ \\
\hline Capim-favorito & Rhynchelytrum repens (Willd.) Hubb. & Gramineae & $\mathrm{Ufla}^{(1)}$ \\
\hline Braquiária decumbens & Brachiaria decumbens Stapf & Gramineae & Embrapa $^{(3)}$ \\
\hline B. decumbens CMM & Brachiaria decumbens Stapf & Gramineae & $\mathrm{CMM}^{(4)}$ \\
\hline B. ruziziensis & Brachiaria ruziziensis Germain Evrard & Gramineae & Embrapa $^{(3)}$ \\
\hline B. brizantha & Brachiaria brizantha Stapf & Gramineae & Embrapa $^{(3)}$ \\
\hline B. humidicola & Brachiaria humidicola (R.) Scheweickt & Gramineae & Embrapa $^{(3)}$ \\
\hline Capim-carrapicho & Cenchrus echinatus L. & Gramineae & $\mathrm{Ufla}^{(1)}$ \\
\hline Aveia-preta & Avena strigosa Schreb & Gramineae & DZO Ufla ${ }^{(5)}$ \\
\hline Milheto & Pennisetum americanum Schumach & Gramineae & DZO Ufla ${ }^{(5)}$ \\
\hline Sorgo & Sorgum sudanensis L. & Gramineae & DZO Ufla ${ }^{(5)}$ \\
\hline Capim-rhodes & Chloris gayana Kunth & Gramineae & DZO Ufla ${ }^{(5)}$ \\
\hline Capim-colonião & Panicum maximum Jacq. cv. Colonião & Gramineae & Embrapa $^{(3)}$ \\
\hline Capim-colonião CMM & Panicum maximum Jacq. cv. Colonião & Gramineae & $\mathrm{CMM}^{(4)}$ \\
\hline Capim-tanzânia & Panicum maximum Jacq. cv. Tanzânia & Gramineae & Embrapa $^{(3)}$ \\
\hline Capim-mombaça & Panicum maximum Jacq. cv. Mombaça & Gramineae & Embrapa $^{(3)}$ \\
\hline Capim-andropogon & Andropogon gayanus Kunt cv. Baeté & Gramineae & Embrapa $^{(3)}$ \\
\hline Capim-andropogon CMM & Andropogon gayanus Kunt cv. Baeté & Gramineae & $\mathrm{CMM}^{(4)}$ \\
\hline Capim-gordura & Melinis minutiflora Beauv & Gramineae & DZO Ufla ${ }^{(5)}$ \\
\hline Capim-setária & Setaria sphacelata Schumach Moss & Gramineae & Embrapa $^{(3)}$ \\
\hline Teosinto & Euchlaena mexicana Schrad & Gramineae & DZO Ufla ${ }^{(5)}$ \\
\hline Capim-urocloa & Urocloa mosambicensis L. & Gramineae & Embrapa $^{(6)}$ \\
\hline Tiririca & Cyperus sp. & Cyperaceae & $\mathrm{Ufla}^{(1)}$ \\
\hline Calaminaceae CMM & Pffafia sp. & Amaranthacea & $\mathrm{CMM}^{(7)}$ \\
\hline Trevo-branco & Trifolium repens $\mathrm{L}$. & Gramineae & DZO Ufla ${ }^{(5)}$ \\
\hline Capim-búfalo & Cenchrus ciliares L. & Gramineae & Embrapa $^{(5)}$ \\
\hline Capim CMM & Paspalum sp. & Gramineae & $\mathrm{CMM}^{(4)}$ \\
\hline Grama-seda & Cynodon dactylon (L.) Pers & Gramineae & $\mathrm{CMM}^{(7)}$ \\
\hline
\end{tabular}

Tabela 2. Concentrações de cádmio e zinco (Mehlich-1) nas misturas de solo com diferentes proporções de solo contaminado estudado.

\begin{tabular}{|c|c|c|c|c|c|c|c|c|}
\hline \multirow{2}{*}{$\begin{array}{l}\text { Metais } \\
\left(\mathrm{mg} \mathrm{dm}^{-3}\right)\end{array}$} & \multicolumn{6}{|c|}{ Proporção de solo contaminado (\%) } & \multirow[t]{2}{*}{ Equação de ajuste $^{(1)}$} & \multirow[t]{2}{*}{$\mathrm{R}^{2}$} \\
\hline & 0 & 7,5 & 15 & 30 & 45 & 60 & & \\
\hline $\mathrm{Zn}$ & 20 & 273 & 456 & 896 & 1.274 & 1.352 & $Y=94,05+23,42 x$ & $0,96^{* *}$ \\
\hline $\mathrm{Cd}$ & $<1$ & 9 & 25 & 49 & 79 & 85 & $Y=1,05+1,53 x$ & $0,97 * *$ \\
\hline
\end{tabular}

${ }^{(1)} \mathrm{Y}$ : concentração de metal no solo; X: proporção de solo contaminado. ${ }^{* *}$ Significativo a $1 \%$ de probabilidade, pelo teste $\mathrm{F}$. 
Os resultados foram submetidos à análise de variância e, com base nas respostas das espécies à contaminação, estimaram-se, pelas regressões, as proporções de solo contaminado necessário para reduzirem $50 \%\left(\mathrm{P}_{50}\right)$ da matéria seca da parte aérea de cada espécie e a concentração de $\mathrm{Cd}$ e Zn no solo nessa proporção.

\section{Resultados e Discussão}

A Pffafia sp. apresentou efeito depressivo na produção de matéria seca da parte aérea (MSPA) a partir de $30 \%$ de solo contaminado, ao passo que A. gayanus CMM foi severamente afetado, não crescendo neste grau de contaminação (Figura 1). Hou-
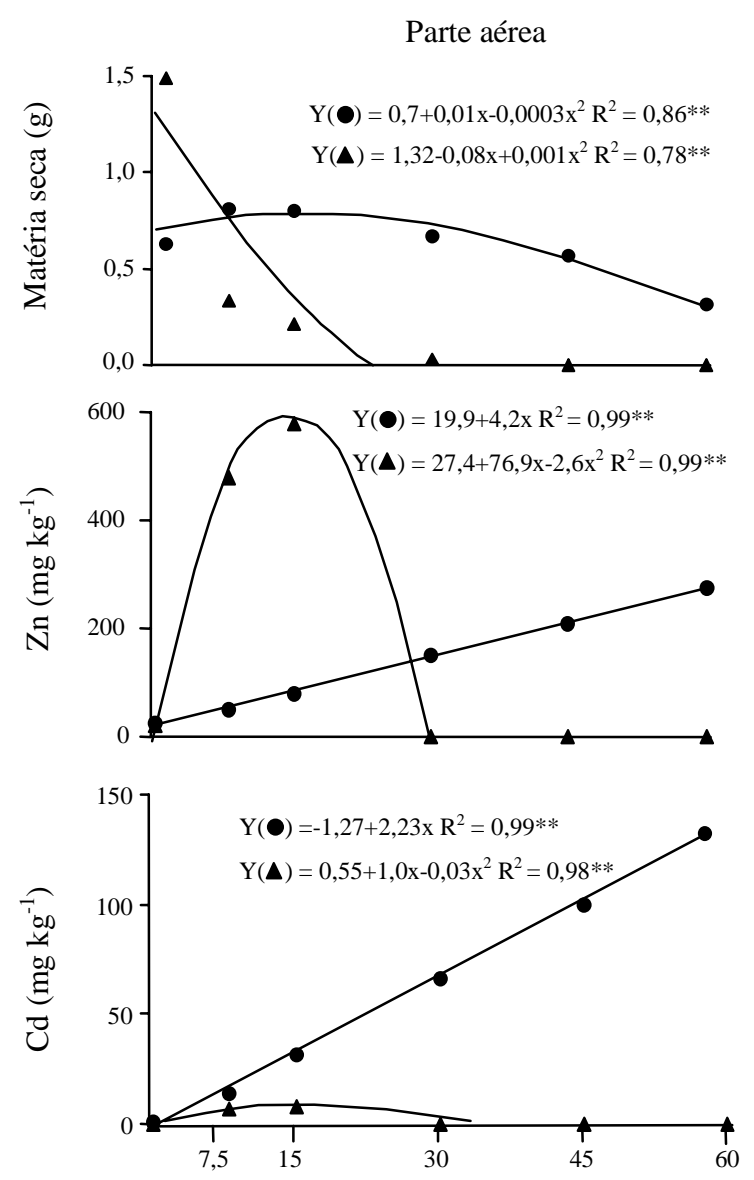

Proporção de solo contaminado (\%) ve forte efeito negativo do aumento da contaminação na produção de matéria seca da parte aérea em todas as espécies estudadas, o qual pode ser constatado pelo sinal negativo do coeficiente " $b$ " da equação de regressão (Tabela 3 ).

As espécies S. glaziovii, B. pilosa, R. repens, C. echinatus e N. physaloides germinaram, emitindo radículas, que em contato com o solo contaminado, mesmo na menor contaminação $(7,5 \%)$, adquiriram coloração escura e posteriormente necrose, com morte da plântula, demonstrando alta sensibilidade à contaminação do solo com metais pesados.
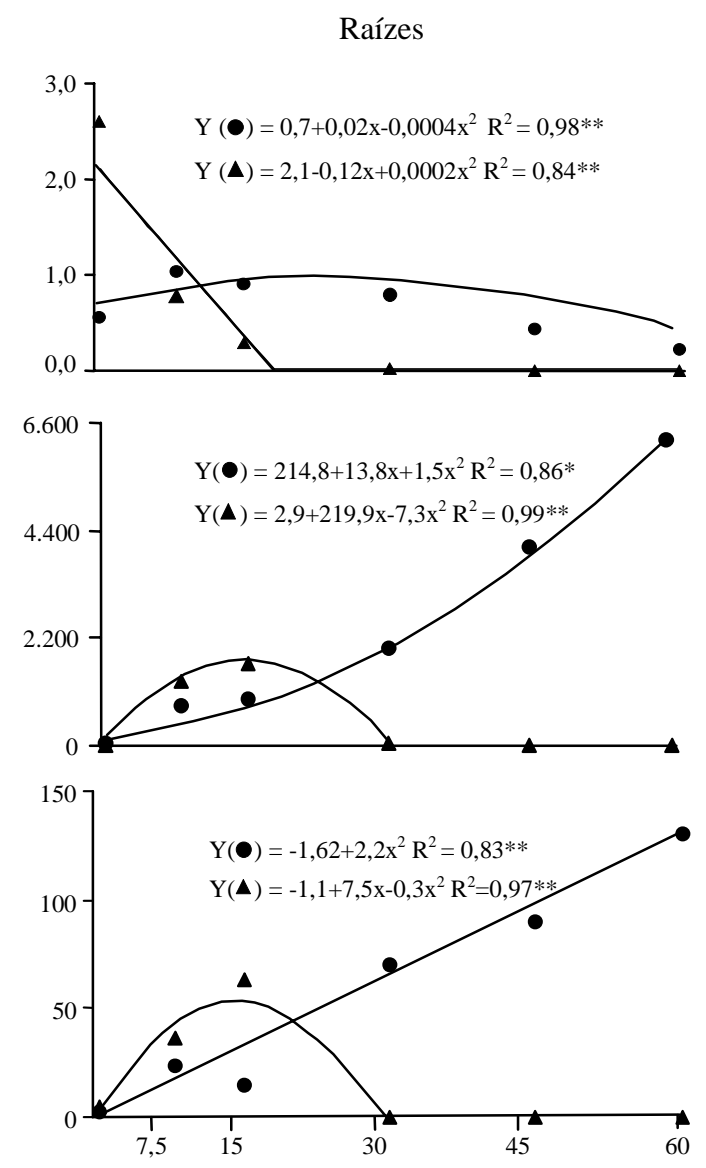

Figura 1. Matéria seca e concentrações de Cd e Zn e da parte aérea e das raízes de Andropogon gayanus CMM (A) e de Pffafia sp. (), em razão da proporção de solo contaminado com metais pesados. * e ** Significativo a $5 \%$ e a $1 \%$, de probabilidade, respectivamente, pelo teste $\mathrm{F}$. 
Os materiais originados de áreas contaminadas, designados CMM, como A. gayanus, P. maximum cv. Colonião, Paspalum sp. e B. decumbens mostraramse sensíveis à contaminação do solo. Essas espécies não ocorrem naturalmente na área de estudo; foram introduzidas no local da fábrica da CMM, em Três Marias, MG, através de plantio, na tentativa recente de revegetar, onde sobrevivem em moitas. Os resultados indicam não estarem adaptadas ao excesso de metais no solo, pois comportam-se de modo semelhante às espécies obtidas de áreas não contaminadas (Tabela 3).

Misturas contendo acima de $15 \%$ de solo contaminado reduziram drasticamente a biomassa de raízes de capim-andropogon (Figura 1). Em Pffafia sp. constatou-se máxima produção de raízes $(0,94 \mathrm{~g})$ na mistura com $30 \%$ de solo, apresentando redução, em relação ao controle, a partir de $50 \%$ de solo contaminado (Figura 1), corroborando os resultados encontrados na MSPA e evidenciando a tolerância dessa espécie aos metais pesados no solo. O impacto que os metais pesados exercem na produção de raízes depende da sensibilidade da planta e da intensidade de contaminação, sendo consideradas espécies tolerantes aquelas que apresentam produção de raízes significativas em solos contaminados (Punz \& Sieghardt, 1993), como evidenciado na Pffafia sp. A produção de raízes é uma característica importante quanto à fitoestabilização de áreas contaminadas com metais pesados, pois protege o solo da erosão, reduz a lixiviação, favorece a agregação e a atividade microbiana do solo.

O comportamento apresentado pela Pffafia sp. pode ser decorrente do fato de a espécie ocorrer naturalmente em áreas de mineração, pois segundo Watanabe (1997), a vegetação encontrada em áreas contaminadas representa importante fonte potencial de ecótipos tolerantes à poluição e apresenta-se adaptada ao ambiente estressante. As plantas tolerantes são endêmicas de solos poluídos, o que sugere significativa adaptação ecofisiológica, manifestando resistência a contaminação do solo com metais pesados (Raskin et al., 1994). Os mecanismos de resistência a metais pesados são vários, como o

Tabela 3. Resposta de espécies herbáceas à elevação de solo contaminado com metais pesados na mistura ${ }^{(1)}$.

\begin{tabular}{|c|c|c|c|c|}
\hline \multirow[t]{2}{*}{ Espécie } & \multicolumn{2}{|c|}{ Matéria seca da parte aérea } & \multicolumn{2}{|c|}{ Matéria seca das raízes } \\
\hline & Equação & $\mathrm{R}^{2}$ & Equação & $\mathrm{R}^{2}$ \\
\hline Brachiaria humidicola & $Y=1,9-0,09 x+0,001 x^{2}$ & $0,96 * *$ & ND & \\
\hline Brachiaria decumbens & $Y=3,1-0,16 x+0,002 x^{2}$ & $0,99 * *$ & $Y=1,7-0,08 x+0,001 x^{2}$ & $0,95 * *$ \\
\hline Brachiaria brizantha & $Y=3,1-0,15 x+0,002 x^{2}$ & $0,97 * *$ & $Y=2,8-0,14 x+0,002 x^{2}$ & $0,97 * *$ \\
\hline Brachiaria ruziziensis & $Y=4,3-0,23 x+0,003 x^{2}$ & $0,92 * *$ & $Y=1,8-0,1 \mathrm{x}+0,001 \mathrm{x}^{2}$ & $0,87 * *$ \\
\hline Brachiaria decumbens CMM & $Y=2,6-0,12 x+0,001 x^{2}$ & $0,75^{* *} *$ & $\mathrm{Y}=2,1-0,1 \mathrm{x}+0,001 \mathrm{x}^{2}$ & $0,79 * *$ \\
\hline Sorghum sudanensis & $Y=2,2-0,1 \mathrm{x}+0,001 \mathrm{x}^{2}$ & $0,90 * *$ & $Y=1,1-0,05 x+0,0006 x^{2}$ & $0,89 * *$ \\
\hline Euchlaena mexicana & $Y=2,5-0,08 x+0,0006 x^{2}$ & $0,99 * *$ & $Y=1,1-0,04 x+0,0004 x^{2}$ & $0,96^{* *}$ \\
\hline Urocloa mosambicensis & $Y=2,5-0,1 x+0,001 x^{2}$ & $0,99 * *$ & $Y=2,3-0,09 x+0,0008 x^{2}$ & $0,98 * *$ \\
\hline Cynodon dactylon & $Y=1,1-0,03 x+0,0001 x^{2}$ & $0,99 * *$ & $Y=0,7-0,02 x+0,0001 x^{2}$ & $0,98 * *$ \\
\hline Andropogon gayanus & $Y=2,1-0,1 x+0,001 x^{2}$ & $0,98 * *$ & $Y=2,7-0,13 x+0,002 x^{2}$ & $0,97 * *$ \\
\hline Panicum maximum cv. Tanzânia & $Y=2,4-0,1 x+0,001 x^{2}$ & $0,98 * *$ & $Y=1,3-0,05 x+0,0006 x^{2}$ & $0,97 * *$ \\
\hline Panicum maximum cv. Mombaça & $Y=2,5-0,1 \mathrm{x}+0,001 \mathrm{x}^{2}$ & $0,96 * *$ & $Y=1,4-0,06 x+0,0006 x^{2}$ & $0,93 * *$ \\
\hline Panicum maximum cv. Colonião & $Y=1,9-0,09 x+0,001 x^{2}$ & $0,95 * *$ & $Y=1,1-0,05 x+0,0005 x^{2}$ & $0,99 * *$ \\
\hline Panicum maximum cv. Colonião CMM & $Y=1,3-0,05 x+0,0004 x^{2}$ & $0,97 * *$ & $Y=1,2-0,05 x+0,0005 x^{2}$ & $0,98 * *$ \\
\hline Arachis pintoi & $Y=1,0-0,05 x+0,0006 x^{2}$ & $0,92 * *$ & $Y=1,7-0,1 x+0,001 x^{2}$ & $0,84 * *$ \\
\hline Setaria sphacelata & $Y=2,3-0,1 x+0,001 x^{2}$ & $0,97 * *$ & $Y=1,6-0,06 x+0,0006 x^{2}$ & $0,98 * *$ \\
\hline Melinis minutiflora & $Y=3,3-0,2 x+0,003 x^{2}$ & $0,82 * *$ & $Y=2,0-0,12 x+0,002 x^{2}$ & $0,82 * *$ \\
\hline Paspalum sp. CMM & $Y=1,0-0,04 x+0,0004 x^{2}$ & $0,99 * *$ & $Y=0,7-0,02 x+0,0002 x^{2}$ & $0,93 * *$ \\
\hline Cenchrus ciliares & $Y=2,2-0,08 x+0,0007 x^{2}$ & $0,96 * *$ & $Y=2,0-0,07 x+0,0005 x^{2}$ & $0,94 * *$ \\
\hline Cyperus sp. & $Y=0,8-0,03 x+0,0003 x^{2}$ & $0,93 * *$ & $Y=3,4-0,12 x+0,001 x^{2}$ & $0,88 * *$ \\
\hline Trifolium repens & $Y=0,6-0,02 x+0,0002 x^{2}$ & $0,96 * *$ & $Y=0,6-0,02 x+0,0002 x^{2}$ & $0,98 * *$ \\
\hline Chloris gayana & $Y=2,1-0,11 x+0,0012 x^{2}$ & $0,91 * *$ & $Y=1,4-0,08 x+0,001 x^{2}$ & $0,81 * *$ \\
\hline Avena strigosa & $Y=1,0-0,04 x+0,0003 x^{2}$ & $0,95 * *$ & $Y=0,2-0,01 x+0,0001 x^{2}$ & $0,81 * *$ \\
\hline Pennisetum americanum & $Y=2,1-0,09 x+0,001 x^{2}$ & $0,98 * *$ & $Y=0,8-0,03 x+0,0002 x^{2}$ & $0,98 * *$ \\
\hline
\end{tabular}

${ }^{(1)}$ Y: matéria seca da parte aérea ou raízes em gramas; X: proporção de solo contaminado; ND: não-determinado. **Significativo a $1 \%$ de probabilidade pelo teste de F. 
impedimento na absorção em conseqüência da alteração da permeabilidade da membrana, mudança na capacidade de absorção, aumento da exsudação de substâncias quelantes e mecanismos bioquímicos alteração nas formas de compartimentalização do metal pesado, alteração no metabolismo celular e na produção intercelular de compostos ligantes (Shaw, 1989; Schat \& Kalff, 1992). Estudos sobre os mecanismos de resistência utilizados por Pffafia sp. na tolerância à contaminação com metais pesados são inexistentes e necessitam ser avaliados.

As concentrações de $\mathrm{Cd}$ e $\mathrm{Zn}$ na parte aérea das plantas aumentaram com a elevação na proporção de solo contaminado, atingindo o valor máximo, na maioria dos casos, com $15 \%$ de solo contaminado na mistura, com exceção daquelas que responderam linearmente à contaminação (Tabela 4). Nessa proporção de contaminação as espécies variaram muito quanto aos teores desses metais, ou seja, de 41 a $5.446 \mathrm{mg} \mathrm{kg}^{-1} \mathrm{de}$ $\mathrm{Zn}$ e de 7 a $94 \mathrm{mg} \mathrm{kg}^{-1}$ de cádmio. As espécies com teores mais elevados de $\mathrm{Zn}$ na parte aérea foram B. humidicola, S. sudanensis, S. sphacelata, T. repens, C. gayana e A. strigosa. Espécies como P. maximum cv. Mombaça, P. maximum cv. Colonião
CMM e $P$. americanum apresentaram concentrações menores que $66 \mathrm{mg} \mathrm{kg}^{-1}$ de zinco. Quanto ao Cd, as concentrações maiores foram verificadas em B. ruziziensis, U. mosambicensis, S. sphacelata, Paspalum sp., C. gayana e A. strigosa.

A. gayanus CMM atingiu em torno de $600 \mathrm{mg} \mathrm{kg}^{-1} \mathrm{de}$ Zn, enquanto a Pffafia sp. não ultrapassou $200 \mathrm{mg} \mathrm{kg}^{-1} \mathrm{de}$ Zn na maior contaminação, embora a resposta a esse elemento tenha sido linear (Figura 1). O A. gayanus CMM revelou baixas concentrações de Cd na MSPA, mas na Pffafia sp. as concentrações desse elemento aumentaram linearmente com a contaminação, atingindo valores superiores a $100 \mathrm{mg} \mathrm{kg}^{-1} \mathrm{de} \mathrm{Cd}$ (Figura 1). Tais resultados indicam que esta planta é hiperacumuladora de $\mathrm{Cd}$, fato ainda não relatado na literatura com relação a este gênero de planta. São raras as espécies hiperacumuladoras de $\mathrm{Cd}$, com registro apenas de Brassica sp. (Salt et al., 1995) e Thlaspi caerulenscens (Cunningham \& Ow, 1996), ambas pertencentes à família Brassicaceae. É interessante o fato de Pffafia sp. ser hiperacumuladora de $\mathrm{Cd}$, mas não de $\mathrm{Zn}$, embora a hiperacumulação de Zn possa ocorrer em concentrações mais elevadas desse elemento no solo do que as concentrações estudadas no presente trabalho.

Tabela 4. Resposta das plantas em relação à concentração de zinco e cádmio na matéria seca da parte aérea e concentração máxima $\left(\mathrm{mg} \mathrm{kg}^{-1}\right)$ desses elementos na mistura com $15 \%$ de solo contaminado $\left(\mathrm{C}_{15}\right)$.

\begin{tabular}{|c|c|c|c|c|c|c|}
\hline \multirow[t]{2}{*}{ Espécie } & \multicolumn{2}{|l|}{$\mathrm{Zn}$} & \multicolumn{4}{|c|}{$\mathrm{Cd}$} \\
\hline & Equação $^{(1)}$ & $\mathrm{R}^{2}$ & $\mathrm{C}_{15}$ & Equação & $\mathrm{R}^{2}$ & $\mathrm{C}_{15}$ \\
\hline Brachiaria humidicola & $Y=-101,4+206,6 x-5,3 x^{2}$ & $0,88^{* *}$ & 1.805 & $\mathrm{Y}=6,89+0,71 \mathrm{x}$ & $0,72 * *$ & 18 \\
\hline Brachiaria decumbens & $Y=-183+128,1 x-4,2 x^{2}$ & $0,58^{*}$ & 794 & $Y=0,17+2,1 x-0,07 x^{2}$ & $0,74^{* *}$ & 16 \\
\hline Brachiaria brizantha & $Y=12,1+119,9 x-2,6 x^{2}$ & $0,99 * *$ & 1.224 & $Y=1,53+1,34 x-0,04 x^{2}$ & 0,97 ** & 13 \\
\hline Brachiaria ruziziensis & $Y=67,9+25,2 x-0,5 x^{2}$ & $0,99 * *$ & 333 & $Y=-3,93+3,16 x$ & $0,94^{* *}$ & 43 \\
\hline Brachiaria decumbens CMM & $Y=62,2+50,02 x$ & $0,85^{* *}$ & 813 & $Y=2,35+0,46 x$ & $0,94 * *$ & 9 \\
\hline Sorghum sudanensis & $Y=-0,4+180,4 x-3,6 x^{2}$ & $0,99 * *$ & 1.896 & $Y=2,16+3,04 x-0,10 x^{2}$ & $0,92 * *$ & 26 \\
\hline Euchlaena mexicana & $Y=-84,9+83,19 x$ & $0,96 * *$ & 1.163 & $Y=-5,68+1,62 x$ & $0,87 * *$ & 19 \\
\hline Urochloa mosambicensis & $Y=-0,7+70,4 x$ & $0,99 * *$ & 1.055 & $Y=0,02+2,25 x$ & $0,99 * *$ & 34 \\
\hline Cynodon dactylon & $Y=4,35+10,9 x$ & $0,90 * *$ & 168 & $Y=0,81+0,39 x$ & $0,98^{* *}$ & 7 \\
\hline Andropogon gayanus & $Y=113,0+78,6 x-2,7 x^{2}$ & $0,99 * *$ & 684 & $Y=3,3+1,20 x-0,04 x^{2}$ & $0,99^{* *}$ & 12 \\
\hline Panicum maximum cv. Tanzânia & $Y=57,6+114,3 x-2,2 x^{2}$ & $0,98 * *$ & 1.277 & $Y=2,63+0,67 x$ & $0,95^{* *}$ & 13 \\
\hline Panicum maximum cv. Mombaça & $Y=14,4+1,74 x$ & $0,99 * *$ & 41 & $\mathrm{Y}=2,17+1,02 \mathrm{x}$ & $0,98 * *$ & 17 \\
\hline Panicum maximum $\mathrm{cv}$. Colonião & $Y=-1,2+114,0 x-1,9 x^{2}$ & $0,98 * *$ & 1.281 & $Y=4,33+0,8 x$ & $0,91 * *$ & 16 \\
\hline Panicum maximum cv. Colonião CMM & $Y=27,0+1,03 x$ & $0,95 * *$ & 42 & $Y=1,12+1,75 x-0,03 x^{2}$ & $0,98^{* *}$ & 21 \\
\hline Arachis pintoi & $Y=101,6+187,1 x-6,4 x^{2}$ & $0,99 * *$ & 1.468 & $Y=0,61+3,5 x-0,12 x^{2}$ & $0,99^{* *}$ & 26 \\
\hline Setaria sphacelata & $Y=210,3+265,4 x-8,8 x^{2}$ & $0,85^{* *}$ & 2.211 & $Y=3,51+9,78 x-0,33 x^{2}$ & $0,91^{* *}$ & 76 \\
\hline Paspalum sp. CMM & $Y=64,5+49,9 x$ & $0,96^{* *}$ & 813 & $Y=0,83+3,06 x-0,04 x^{2}$ & $0,99^{* *}$ & 38 \\
\hline Cenchrus ciliares & $Y=88,3+48,2 x$ & $0,99 * *$ & 811 & $Y=6,41+0,74 x$ & $0,77 * *$ & 18 \\
\hline Cyperus sp. & $Y=17,2+106,3 x-3,5 x^{2}$ & $0,94 * *$ & 824 & $Y=-1,33+2,34 x-0,07 x^{2}$ & $0,83^{* *}$ & 18 \\
\hline Trifolium repens & $Y=-715,8+753,2 x-24,0 x^{2}$ & $0,74 * *$ & 5.182 & $Y=1,73+3,47 x-0,12 x^{2}$ & $0,98^{* *}$ & 27 \\
\hline Chloris gayana & $Y=-159,4+739,7 x-24,4 x^{2}$ & $0,97^{* *}$ & 5.446 & $Y=-3,12+9,63 x-0,32 x^{2}$ & $0,94^{* *}$ & 69 \\
\hline Avena strigosa & $Y=49,2+221,4 x-7,4 x^{2}$ & $0,97 * *$ & 1.705 & $Y=-2,28+12,55 x-0,41 x^{2}$ & $0,94^{* *}$ & 94 \\
\hline Pennisetum americanum & $\mathrm{Y}=34,3+2,1 \mathrm{x}+0,003 \mathrm{x}^{2}$ & $0,97 * *$ & 66 & $\mathrm{Y}=4,5+2,73 \mathrm{x}$ & $0,98^{* *}$ & 45 \\
\hline
\end{tabular}

${ }^{(1)} \mathrm{Y}$ : concentrações de $\mathrm{Zn}$ e $\mathrm{Cd}\left(\mathrm{mg} \mathrm{kg}^{-1}\right)$ na matéria seca da parte aérea; X: proporção de solo contaminado. * e**Significativo a $5 \%$ e a $1 \%$ de probabilidade, respectivamente, pelo teste $\mathrm{F}$. 
As concentrações de $\mathrm{Cd}$ e Zn na matéria seca das raízes apresentaram comportamento bastante semelhante ao verificado em relação a MSPA (Tabela 5). B. humidicola, A. pintoi, M. minutiflora, C. gayana e A. strigosa apresentaram baixa produção de matéria seca de raízes, o que impossibilitou a realização de análises dos teores desses elementos nos tecidos. Em geral, as concentrações de $\mathrm{Zn}$ e Cd nas raízes atingiram o máximo na proporção de $15 \%$ de solo contaminado na maioria das espécies estudadas, com exceção daquelas que apresentaram respostas lineares (Tabela 5). Nesse nível de contaminação, as espécies C. dactylon, Paspalum sp., C. ciliares e Cyperus sp. apresentaram menores concentrações de Zn, variando de $294 \mathrm{mg} \mathrm{kg}^{-1}$ em Paspalum sp. a $992 \mathrm{mg} \mathrm{kg}^{-1} \mathrm{em} \mathrm{C}$. dactylon. As demais espécies apresentaram elevada concentração desse elemento, com valores superiores a $3.000 \mathrm{mg} \mathrm{kg}^{-1} \mathrm{em} \mathrm{A}$. gayanus, S. sudanensis, S. sphacelata e T. repens. As concentrações de $\mathrm{Cd}$ nas raízes variaram de $18 \mathrm{mg} \mathrm{kg}^{-1}$ em Cyperus sp. até $348 \mathrm{mg} \mathrm{kg}^{-1} \mathrm{em}$ T. repens. Em relação a Pffafia sp., as concentrações de $\mathrm{Zn}$ foram crescentes com aumento da contaminação do solo com metais pesados, enquanto em
A. gayanus CMM a máxima concentração foi alcançada na proporção de $15 \%$ de solo contaminado, decrescendo com o aumento da contaminação (Figura 1). Quanto ao Cd nas raízes de Pffafia sp., o comportamento foi semelhante ao encontrado na MSPA, inclusive em termos de valores, indicando a elevada translocação desse elemento nesta espécie (Figura 1). A concentração de $\mathrm{Zn}$ nas raízes de Pffafia sp. foi elevada em relação à concentração na MSPA. Essa retenção de metais pesados nas raízes de Pffafia sp. pode estar relacionada a um mecanismo de tolerância ao excesso de $\mathrm{Zn}$, como verificado em outras espécies tolerantes (Marchiol et al., 1996; Schat \& Verkleij, 1998).

Para discriminar melhor o comportamento das espécies, estimou-se, por regressões, a proporção de solo contaminado necessário para reduzir em $50 \%\left(\mathrm{P}_{50}\right)$ a matéria seca da parte aérea das plantas (Tabela 3). Esse índice mostra a influência que a contaminação exerce sobre as plantas (Soares, 1999) e sobre as atividades biológicas do solo (Speir et al., 1995; Moreno et al., 2001), sendo considerado indicativo de tolerância/sensibilidade. Nesse contexto, houve variação de apenas $6 \%$ para A. gayanus

Tabela 5. Resposta das plantas para concentração de zinco e cádmio na matéria seca das raízes e concentração máxima $\left(\mathrm{mg} \mathrm{kg}^{-1}\right)$ desses elementos na mistura com $15 \%$ de solo contaminado $\left(\mathrm{C}_{15}\right)$.

\begin{tabular}{|c|c|c|c|c|c|c|}
\hline \multirow[t]{2}{*}{ Espécie } & \multicolumn{3}{|c|}{$\mathrm{Zn}$} & \multicolumn{3}{|l|}{$\mathrm{Cd}$} \\
\hline & Equação $^{(1)}$ & $\mathrm{R}^{2}$ & $\mathrm{C}_{15}$ & Equação & $\mathrm{R}^{2}$ & $\mathrm{C}_{15}$ \\
\hline Brachiaria decumbens & $Y=-243,4+388,6 x-12,6 x^{2}$ & $0,87^{* *}$ & 2.751 & $Y=-3,38+13,0 x-0,43 x^{2}$ & $0,95 * *$ & 95 \\
\hline Brachiaria brizantha & $Y=-37,9+234,8 x-7,7 x^{2}$ & $0,84 * *$ & 1.751 & $Y=-1,55+8,8 x$ & $0,97 * *$ & 130 \\
\hline Brachiaria ruziziensis & $Y=44,8+210,8 x-7,1 x^{2}$ & $0,99 * *$ & 1.609 & $Y=-7,0+13,3 x-0,43 x^{2}$ & $0,91 * *$ & 96 \\
\hline Brachiaria decumbens $\mathrm{CMM}$ & $Y=-104,6+193,3 x-6,3 x^{2}$ & $0,88^{* *}$ & 1.377 & $Y=-2,9+6,2 x-0,2 x^{2}$ & $0,92 * *$ & 45 \\
\hline Sorghum sudanensis & $Y=-98,8+491,5 x-15,2 x^{2}$ & $0,95^{* *}$ & 3.853 & $Y=-6,8+12,9 x-0,42 x^{2}$ & $0,89 * *$ & 92 \\
\hline Euchlaena mexicana & $Y=132,2+242,2 x-6,5 x^{2}$ & $0,99 * *$ & 2.302 & $\mathrm{Y}=5,2+5,8 \mathrm{x}-0,2 \mathrm{x}^{2}$ & $0,87 * *$ & 47 \\
\hline Urochloa mosambicensis & $Y=-27,6+137,7 x-0,79 x^{2}$ & $0,99 * *$ & 1.860 & $Y=-1,44+3,24 x$ & $0,99 * *$ & 47 \\
\hline Cynodon dactylon & $\mathrm{Y}=34,5+67,9 \mathrm{x}-0,27 \mathrm{x}^{2}$ & $0,99 * *$ & 992 & $Y=2,76+1,6 x$ & $0,97 * *$ & 28 \\
\hline Andropogon gayanus & $Y=-46,1+429,8 x-14,2 x^{2}$ & $0,98^{* *}$ & 3.205 & $Y=-12,4+17,4 x-0,56 x^{2}$ & $0,86^{* *}$ & 123 \\
\hline Panicum maximum cv. Tanzânia & $Y=420,6+223,46 x-3,53 x^{2}$ & $0,98 * *$ & 2.979 & $Y=-6,45+8,58 x$ & $0,99 * *$ & 122 \\
\hline Panicum maximum cv. Mombaça & $Y=118,9+213,1 x-2,8 x^{2}$ & $0,98 * *$ & 2.685 & $Y=0,69+6,7 x$ & $0,99 * *$ & 100 \\
\hline Panicum maximum cv. Colonião & $Y=47,3+203,8 x-3,9 x^{2}$ & $0,99 * *$ & 2.226 & $Y=4,24+8,9 x-0,18 x^{2}$ & $0,88^{* *}$ & 97 \\
\hline Panicum maximum cv. Colonião CMM & $Y=133,0+304,53 x-10,3 x^{2}$ & $0,98 * *$ & 2.383 & $Y=0,85+13,8 x-0,46 x^{2}$ & $0,99 * *$ & 104 \\
\hline Setaria sphacelata & $Y=185,3+330,4 x-6,7 x^{2}$ & $0,98^{* *}$ & 3.633 & $Y=6,7+12,6 x-0,21 x^{2}$ & $0,98 * *$ & 148 \\
\hline Paspalum sp. CMM & $Y=124,3+21,8 x-0,7 x^{2}$ & $0,98^{* *}$ & 294 & $Y=-7,6+20,4 x-0,67 x^{2}$ & $0,92 * *$ & 148 \\
\hline Cenchrus ciliares & $Y=85,9+24,6 x+1,6 x^{2}$ & $0,98 * *$ & 815 & $Y=0,84+1,74 x$ & $0,97 * *$ & 27 \\
\hline Cyperus sp. & $Y=11,3+76,3 x-0,86 x^{2}$ & $0,97^{* *}$ & 962 & $Y=2,21+0,97 x$ & $0,94 * *$ & 17 \\
\hline Trifolium repens & $Y=362,1+439,2 x-13,1 x^{2}$ & $0,94 * *$ & 4.003 & $Y=17,7+44,8 x-1,52 x^{2}$ & $0,87^{* *}$ & 348 \\
\hline Pennisetum americanum & $Y=159,0+315,6 x-10,7 x^{2}$ & $0,99 * *$ & 2.486 & $Y=0,03+4,49 x-0,15 x^{2}$ & $0,99 * *$ & 34 \\
\hline Arachis pintoi & Sem ajuste & & & Sem ajuste & & \\
\hline Melinis minutiflora & Sem ajuste & & & Sem ajuste & & \\
\hline Chloris gayana & Sem ajuste & & & Sem ajuste & & \\
\hline Avena strigosa & Sem ajuste & & & Sem ajuste & & \\
\hline
\end{tabular}

${ }^{(1)} \mathrm{Y}$ : concentrações de $\mathrm{Zn}$ e $\mathrm{Cd}\left(\mathrm{mg} \mathrm{kg}^{-1}\right)$ na matéria seca das raízes; X: proporção de solo contaminado. **Significativo a $1 \%$ de probabilidade pelo teste F. 
CMM e 58\% para Pffafia sp. (Figura 2), indicando a elevada sensibilidade da primeira, a tolerância da última e posições intermediárias das demais espécies estudadas. As espécies E. mexicana, C. dactylon, Cyperus sp. e T. repens apresentaram $\mathrm{P}_{50}$ na faixa de $15 \%$ a $20 \%$ de solo contaminado, ao passo que em B. ruziziensis, A. gayanus CMM, A. pintoi e M. minutiflora os valores de $\mathrm{P}_{50}$ foram inferiores a $10 \%$. No primeiro grupo de plantas $\left(\mathrm{P}_{50}\right.$ de $15 \%$ a $20 \%$ ) as concentrações de $\mathrm{Cd}$ e $\mathrm{Zn}$ no solo capazes de reduzir $50 \%$ da MSPA foram estimadas, com base na regressão (Tabela 2), em 32 e $562 \mathrm{mg} \mathrm{kg}^{-1}$, respectivamente; em relação ao segundo grupo $\left(\mathrm{P}_{50}<10 \%\right)$ foram de $16 \mathrm{mg} \mathrm{kg}^{-1}$ de Cde $328 \mathrm{mg} \mathrm{kg}^{-1}$ de Zn (Figu- ra 2). Quanto a Pffafia sp., somente concentrações elevadas (90 $\mathrm{mg} \mathrm{kg}^{-1}$ de Cd e de $1.450 \mathrm{mg} \mathrm{kg}^{-1} \mathrm{de} \mathrm{Zn}$ ) inibiram a produção da matéria seca da parte aérea em $50 \%$.

Considerando que o nível crítico de $\mathrm{Cd}$ e $\mathrm{Zn}$ no solo, no qual o crescimento da planta é reduzido, é de $8 \mathrm{mg} \mathrm{kg}^{-1}$ de Cd e $450 \mathrm{mg} \mathrm{kg}^{-1}$ de $\mathrm{Zn}$ (KabataPendias \& Pendias, 1984), observa-se que o nível crítico de $\mathrm{Zn}$ foi suficiente para promover redução de 50\% na MSPA na maioria das espécies estudadas, com exceção da Pffafia sp., T. repens, E. mexicana, C. dactylon e do Cyperus sp. Apesar de estes autores trabalharem com níveis críticos com base na redução de $10 \%$ da MSPA, verificou-se nes-

Níveis críticos no solo

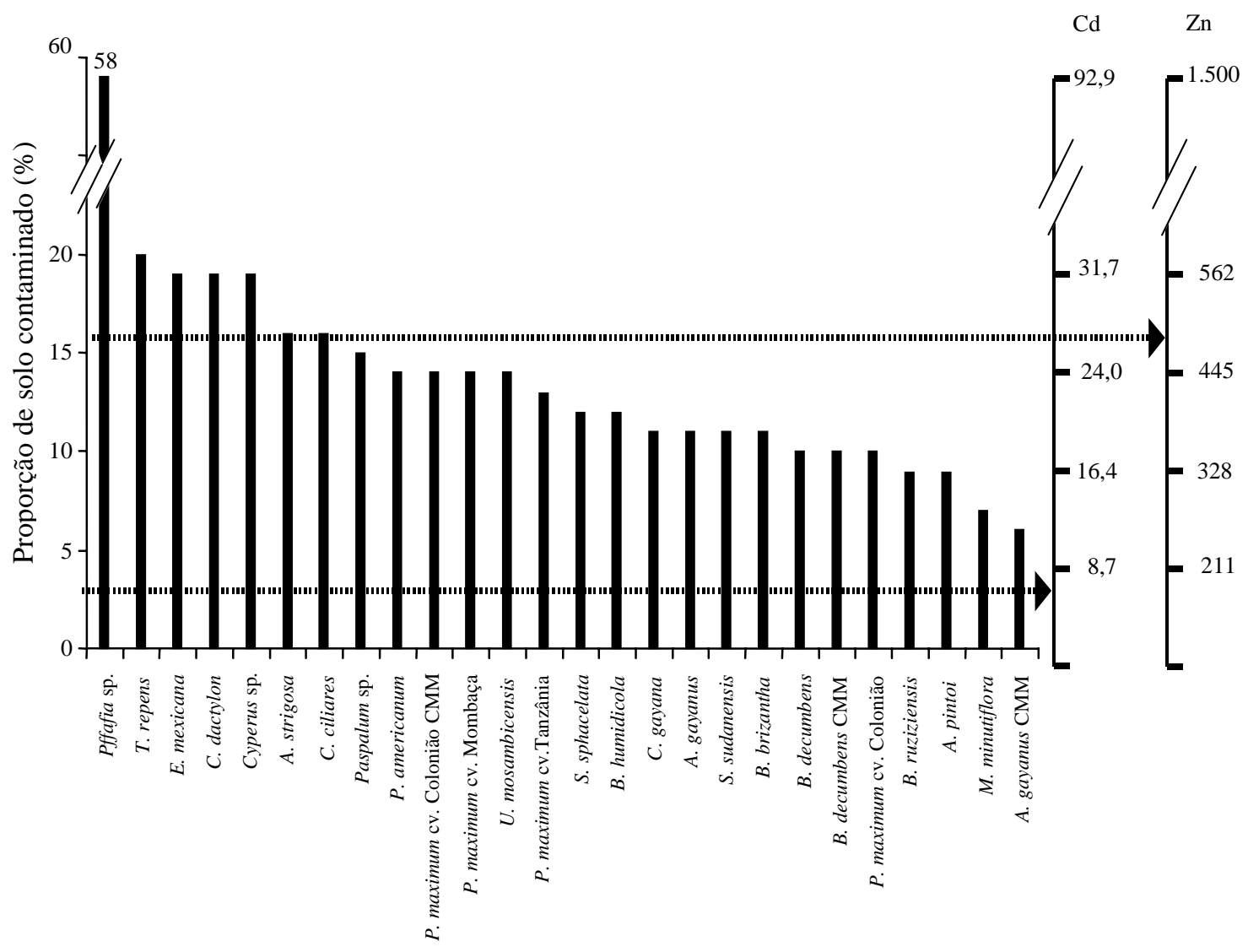

Espécies vegetais

Figura 2. Proporção de solo contaminado com metais pesados necessária para reduzir $50 \%$ da matéria seca da parte aérea das espécies estudadas e níveis críticos $\left(\mathrm{mg} \mathrm{kg}^{-1}\right)$ de $\mathrm{Cd}$ e $\mathrm{Zn}$ no solo (linhas tracejadas). 
te estudo que o nível crítico proposto para Zn no solo está superestimado, o que sugere a necessidade de estudos mais aprofundados envolvendo solos e espécies tropicais.

Baseando-se nos resultados do presente trabalho, apenas Pffafia sp. seria promissora na revegetação de solos contaminados, pois, além de apresentar-se tolerante, é hiperacumuladora de cádmio. No entanto, esta planta apresenta baixa produção de biomassa, como pode ser observado pelo valor da interseção da equação de regressão (Figura 1), o que representa certa desvantagem para a fitorremediação. Tal característica é verificada em outras espécies consideradas acumuladoras de metais pesados (Raskin et al., 1994; Salt et al., 1998). Outras espécies, ainda que menos tolerantes que Pffafia sp., como T. repens, E. mexicana, C. dactylon, Cyperus sp., A. strigosa e $C$. ciliares, são promissoras para a revegetação de áreas contaminadas com metais pesados, quando se faz a diluição ou substituição do solo poluído, ou se emprega agentes amenizantes que reduzem a disponibilidade dos metais no solo, ou ainda o plantio consorciado utilizando estas espécies. Portanto, a escolha de espécies de plantas capazes de revegetar a área contaminada é o primeiro passo para o sucesso da recuperação desse tipo de solo.

\section{Conclusões}

1. As espécies comportam-se de modo diferenciado em relação à contaminação do solo por $\mathrm{Cd} \mathrm{e} \mathrm{Zn}$, variando de espécies tolerantes a muito sensíveis a esses elementos.

2. Pffafia sp. apresenta elevada tolerância à contaminação do solo com Cd e Zn; cresce satisfatoriamente em solo contendo até $90 \mathrm{mg} \mathrm{kg}^{-1}$ de Cd e de $1.450 \mathrm{mg} \mathrm{kg}^{-1}$ de $\mathrm{Zn}$ e contém teores de Cd na matéria seca da parte aérea e das raízes superiores a $100 \mathrm{mg} \mathrm{kg}^{-1}$, sendo, por isso, considerada hiperacumuladora de cádmio.

3. As espécies Sida glaziovii, Bidens pilosa, Rhynchelytrum repens, Cenchrus echinatus e Nicandra physaloides são extremamente sensíveis à contaminação do solo com $\mathrm{Cd}$ e zinco.

\section{Referências}

ACCIOLY, A. M. A. Amenizantes e estratégias para estabelecimento de vegetação em solos de áreas contaminadas por metais pesados. 2001. $186 \mathrm{f}$. Tese (Doutorado em Solos e Nutrição de Plantas) - Universidade Federal de Lavras, Lavras, 2001.

ACCIOLY, A. M. A.; SIQUEIRA, J. O. Contaminação química e biorremediação do solo. In: NOVAES, R. F.; ALVAREZ, V. H. V.; SCHAEFER, C. E. G. R. (Ed.). Tópicos em ciência do solo. Viçosa, MG: UFV, 2000. p. 299352.

BAKER, A. J. M. Metal tolerance. New Phytologist, London, v. 106, p. 93-111, 1987.

BAKER, A. J. M.; McGRATH, S. P.; SODOLI, C. M. D.; REEVES, R. D. The possibility of in situ heavy metal decontamination of polluted soils using crops of metalaccumulating plants. Resources, Conservation and Recycling, Amsterdam, v. 11, p. 41-49, 1994.

CARNEIRO, M. A. C.; SIQUEIRA, J. O.; MOREIRA, F. M. S. Estabelecimento de plantas herbáceas em solo contaminado com metais pesados: efeito de misturas de espécies e inoculação com fungos micorrízicos. Pesquisa Agropecuária Brasileira, Brasília, v. 36, n. 12, p. 14431452, dez. 2001.

CUNNINGHAM, S. D.; ANDERSON, T. A.; SCHWAB, A. P.; HSU, F. C. Phytoremediation of soils contaminated with organic pollutants. Advance in Agronomy, New York, v. 56, p. 55-114, 1996.

CUNNINGHAM, S. D.; OW, D. W. Promises and prospects of phytoremediation. Plant Physiology, Rockville, v. 110, p. 715-719, 1996.

EBBS, S. D.; KOCHIAN, L. V. Phytoextraction of zinc by Oat (Avena sativa), Barley (Hordeum vulgare), and Indian Mustard (Brassica juncea). Environmental Science \& Technology, Washington, v. 32, p. 802-806, 1998.

ELTROP, L.; BROWN, G.; JOACHIM, O.; BRINKMANN, K. Lead tolerance of betula and salix in the mining area of Mechernich/Germany. Plant and Soil, Dordrecht, v. 131, n. 2, p. 279-285, 1991.

FORSTER, J. C. Heavy metals. In: ALEF, K.; NANNIPIERI, P. (Ed.). Methods in applied soil microbiology and biochemistry. London: Academic, 1995. p. 1010-1043. 
HUNTER, A. H. Laboratory analysis of vegetal tissues samples. Raleigh: North Carolina University, 1975. 5 p.

KABATA-PENDIAS, A.; PENDIAS, H. Trace elements in soils and plants. Boca Raton: CRC Press, 1984.315 p.

MARCHIOL, L.; LEITA, L.; MARTIN, M.; PERESSOTTI, A.; ZERBI, G. Physiological responses of two soybean cultivars to cadmium. Journal of Environmental Quality, Madison, v. 25, p. 562-566, 1996.

MORENO, J. L.; GARCIA, C.; LANDI, L.; FALCHINI, L.; PIETRAMELLARA, G.; NANNIPIERI, P. The ecological dose value $\left(\mathrm{ED}_{50}\right)$ for assessing toxicity on ATP content and dehydrogenase and urease activities of soil. Soil Biology \& Biochemistry, Oxford, v. 33, p. 483-489, 2001.

PUNZ, W. F.; SIEGHARDT, H. The response of roots of herbaceous plant species to heavy metals. Environmental and Experimental Botany, Elmsford, v. 44, n. 1, p. 8598, 1993.

RASKIN, I.; KUMAR, P. B. A. N.; DUSHENKOV, S.; SALT, D. E. Bioconcentration of heavy metals by plants. Current Opinion Biotechnology, London, v. 5, p. 285 290, 1994.

SALT, D. E.; BLAYLOCK, M.; KUMAR, N. P. B. A.; DUSHENKOV, V.; ENSLEY, B. D.; CHET, I.; RASKIN, I. Phytoremediaton: a novel strategy for the removal of toxic metals from the environment using plants. Biotechnology, Frankfurt, v. 13, p. 468-474, 1995.

SALT, D. E.; SMITH, R. D.; RASKIN, I. Phytoremediation. Annual Review of Plant Physiology, Palo Alto, v. 49, p. 643-668, 1998.
SCHAT, H.; KALFF, M. M. A. Are phytochelatins involved in differential metal tolerance or do they merely reflect metal-imposed strain? Plant Physiology, Rockville, v. 99, n. 4, p. 1475-1480, 1992.

SCHAT, H.; VERKLEIJ, A. C. Biological interactions: the role for non-woody plant in phytorestoration: possibilities to exploit adaptive heavy metal tolerance. In: VANGRONSVELD, J.; CUNNINGHAM, S. D. (Ed.). Metal-contaminated soils: in situ inactivation and phytorestoration. Berlin: Springer, 1998. p. 51-63.

SENGUPTA, M. Environmental impacts of mining: monitoring, restoration, and control. Boca Raton: Lewis, 1993. $494 \mathrm{p}$.

SHAW, A. J. Heavy metal tolerance in plants: evolutionary aspects. New York: CRC Press, 1989. 355 p.

SOARES, C. R. F. S. Toxidez de zinco, cobre, cádmio e chumbo para o eucalipto em solução nutritiva. 1999. 132 f. Dissertação (Mestrado em Solos e Nutrição de Plantas) - Universidade Federal de Lavras, Lavras, 1999.

SPEIR, T. W.; KETTLES, H. A.; PARSHOTAM, A.; SEARLE, P. L.; VLAAR, L. N. C. Simple kinetic approach to derive the ecological dose value, $\mathrm{ED}_{50}$, for the assessment of $\mathrm{Cr}$ (VI) toxicity to soil biological properties. Soil Biology \& Biochemistry, Oxford, v. 27, p. 801-810, 1995.

VANGRONSVELD, J.; COLPAERT, J. V.; TICHELEN, K. K. van. Reclamation of a bare industrial area contaminated by non-ferrous metals: physicochemical and biological evaluation of the durability of soil treatment and revegetation. Environmental Pollution, Oxford, v. 94, p. 131-140, 1997.

WATANABE, M. E. Phytoremediation on the brink of commercialization. Environmental Science \& Technology, Washington, v. 31, p. 182-186, 1997. 